\title{
Provisioning of Multimedia Services over Open NGN Testbed
}

\author{
Adel Al-Hezmi \\ Fraunhofer FOKUS \\ Berlin, Germany \\ Adel.Al- \\ Hezmi@fokus.fraunhofer.de
}

\author{
Fabricio Carvalho de Gouveia \\ Fraunhofer FOKUS \\ Berlin, Germany \\ Fabricio.Gouveia@fokus.fraun \\ hofer.de
}

\author{
Thomas Magedanz \\ Fraunhofer FOKUS
}

Berlin, Germany
Thomas.magedanz@fokus.fra
unhofer.de

\begin{abstract}
Provisioning of multimedia services over next generation network and across heterogeneous fixed and mobile networks challenges the developers to define a unified triple play framework solution. However the IP Multimedia Subsystem (IMS) defined by the 3rd Generation Partnership Projects (3GPP and 3GPP2) represents today a basis framework that enables the vision of providing triple play services with quality of service and mobility support across heterogeneous networks. The Fraunhofer Institute FOKUS has recently launched the FOKUS Media Interoperability Lab (MIL) that is based on the "Open IMS Playground" testbed. MIL is as an open environment gathering all major IMS core components, and triple play toolkit originating from own developments as well as major industry players, which can be used by academic and industrial partners for early prototyping of new triple play services, related components, protocols, and applications. This paper introduces the relevant technologies and provides an overview of the testbed architecture and the relevant multimedia services at FOKUS. The validation of this architecture is demonstrated with an implementation in the testbed.
\end{abstract}

\section{Keywords}

Triple Play; IMS; TISPAN; Next Generation Networks; Multimedia Applications; SIP; Testbed;

\section{INTRODUCTION}

The IP Multimedia Subsystem (IMS) is an IP network defined by the Third Generation Partnership Project (3GPP) for mobile networks of third generation. It was created given the necessity to bring internet services and session control to the telecom environment and also with the tendency of changing Circuit Switched (CS) networks to Packet Switch (PS) for allowing fast access to the data networks and to services that these offer. This convergence is also referenced to Fixed Mobile Convergence (FMC).

In this context, the idea is to adopt IMS as a platform for operating on top of heterogeneous network environment, consisted of existing fixed and mobile network access technologies, like Wireless Local Area Network (WLAN), Universal Mobile Telecommunications System (UMTS), Digital Subscriber Line (DSL) and many others.

The structure of this paper is as follows: section 2 gives a brief technology background of the important technologies considered in our approach. Section 3 introduces the proposed architecture of an IMS-based interactive streaming framework. Section 4 illustrates the implemented IMS-based hybrid streaming application over UMTS and DVB-H access network. The last section presents conclusions and future outlook.

\section{ELATED WORK AND TECHNOLOGY BACKGROUND}

This section describes briefly the rationale of the main technologies that are considered for the design of the multimedia architecture, describes important considerations in the IMS and within standardization bodies.

\subsection{IMS within the Standardization Bodies}

While there are definitions from the ITU-T and ETSI TISPAN existing for a Next Generation Network (NGN), it essentially is about the convergence of various networks towards an architecture that is based upon the Internet Protocol. From an operator's perspective, the IP Multimedia Subsystem (IMS) is the central point within this architecture to offer service in a secure, controllable, and chargeable and QoS ensured manner. Specified by 3 GPP [1] and 3GPP2 [2], the IMS is now providing also the central elements around which the ETSI TISPAN workgroup is defining its NGN architecture. In a simplified manner, the NGN approach may be represented as a horizontally layered architecture which allows to transport services to users just like traditional network designs (e.g. in ISDN networks), yet it defines service-related functions to be unrelated from underlying technologies and accessible over defined interfaces.

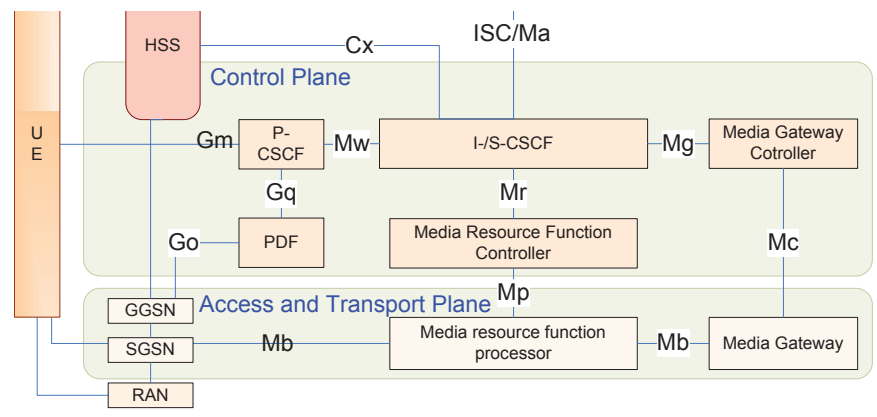

Figure 1: IMS logical layered architecture

For using a service a user is no longer tied to specific access networks, any packet based access network technology may be used. The access networks offer the connection to infrastructures 
in the service control layer like the IMS for a secure and QoS aware access to SIP services or the Internet on a best effort basis. A detailed description of the components can be found in [3] Figure 1 shows the layered approach of the IMS architecture. In spite of the fact that IMS is built on point-to-point communication (unicast) model 3GPP has recently specified in release 6 the Multimedia Broadcast/Multicast Services (MBMS) [4] that enables cellular network to support multicast and broadcast transmission. Although both IMS and MBMS have similar functions such as authentication, authorization and QoS procedures, the specification does not mentioned the integration of both subsystems.

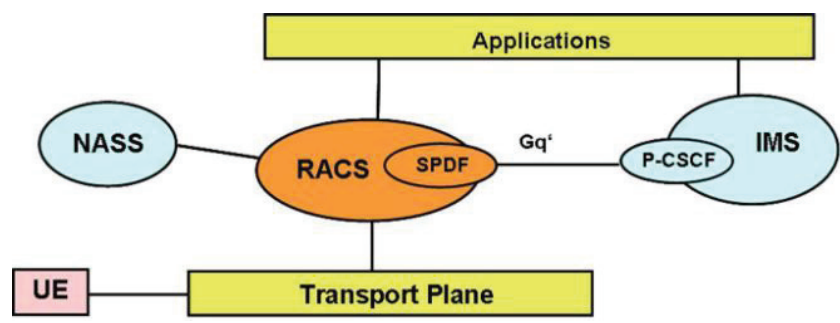

Figure 2: IMS Interface to the TISPAN basic architecture

While 3GPP IMS focuses on mobile world, the reference European standardization body for fixed networks is TISPAN [3]. In its NGN architecture it presents a generic multi-service, multiprotocol, multi-access IP-based framework that aims at becoming the reference model to achieve convergence between PSTN and IP data networks. This model is based upon the concept of cooperating subsystems sharing common components. This architecture enables smooth addition of new subsystems to cover new demands and service classes, and ensures maximum common usage of network resources, applications and user equipment. One of the key subsystems is based on 3GPP IMS Release 6, and it is envisaged to support the provision of SIP-based multimedia services to NGN terminals. TISPAN is currently working on the release 2 with the objective of enabling streaming services (content delivery services like IPTV, streaming, VoD) over NGN with optimized network usage. It is scheduled to be wholly defined throughout 2007. Figure 2 shows the TISPAN reference architecture. This architecture is explained in detail in [5].

\subsection{Related Implementations using the Open IMS Playground}

The University of Cape Town (UCT) in South Africa is making use of the Open Source IMS Toolkit in order to set up their testbeds. They already benefit from the use of the Toolkit [6], where they designed a system to enable human intelligence in the network, which may be realized within the IP multimedia subsystem testbed. UCT also developed an open source IMS client (the UCTIMSClient [7]) that was designed to operate exclusively with the Open Source IMS Core.

These efforts aim to support further research into the specifics of IMS signaling and allow developers a means of testing and designing IMS entities, specifically Application Servers. There are still many promising areas of research like Triple Play and the establishment of testbed federation, which stimulate collaborations.
FOKUS is also developing Similarly extensions to the Digital Video Broadcasting (DVB), which is designed for broadcasting TV services [8]. DVB-T (Terrestrial) is mainly targeted for stationary receivers and is not suitable for mobile devices. To overcome this limitation DVB-H (Handheld) was proposed, which enhanced the physical and link layers of DVB-T to reduce power consumption and improve performance in urban indoor environments. It uses IP protocol for streaming and thus might support multicast transmission in addition to the initial broadcast mode.

\section{FOKUS MEDIA INTEROPERABILITY LAB ARCHITECTURE}

The key challenges of providing IMS-based multimedia services over fixed and mobile networks are the management of the adaptability, mobility, interactivity, context-awareness and personalization. To enable this vision a basic set of common service functions has to be identified. These service functions can be distinguished into three categories: (1) The Content Management Function related to all processing tasks performed during content producing, editing and storing; (2) The Content Delivery Function that manages media processing and content transmission from the source to the end-user with an efficient utilization of available network resources including QoS and mobility support; (3) The Service Control Function that is in charge of the whole life cycle of a service based on a defined business model. This includes session management, access control, service composition, personalization, charging, processing and managing of the interactive application. On the other hand, several players are involved with the value chain of provisioning multimedia services, i.e. (1) the CP who creates, owns or is licensed to sell content; (2) the Service Provider who places the contract with the costumer for supplying personalized multimedia services based on the network capabilities composed by the network delivery system; (3) the Network Delivery System which is in charge of session management and media delivery; (4) the End-User (consumer) domain where multimedia services are consumed.

Provisioning of end-to-end triple play multimedia services with mobility support and quality of service require a cooperated service framework for interworking all players involved in the delivery process. This will imply new requirements on such framework and rise new challenges. These players have to interact smoothly to fulfill the task to provide a whole new service experience to the end user. These requirements were derived from an abstract view to all these players [9].

As TISPAN NGN is based upon the concept of cooperating subsystems sharing common components and providing a framework for an end-to-end all converged network, it will act as the reference architecture for such cooperated framework. Building upon the capabilities of 3GPP IMS as an overlay control subsystem designed to support heterogeneous IP networks and its ability to deliver integrated voice and data services while providing seamless mobility, it will be used as the foundation convergence platform. As discussed in previous section, TISPAN has also standardized IMS in its reference architecture. However IMS is based on unicast transmission mode and thus it has to be enhanced to make use of multicast and broadcast capabilities that are supported by MBMS, DVB-T/H or IP-Multicast. Integration with 3GPP MBMS and DVB-H will bridge this gap in $3 \mathrm{G}$ 
networks and thus enable IMS to be extended to deliver multimedia services efficiently.

During designing process of the FOKUS Media Interoperability Lab (MIL) for enabling the delivery of triple play services all these issues have been taken into consideration. Figure 3 shows the high level logical architecture of the lab that comprises seven planes as follow:

Access and Transport Plane corresponds to the access and core network. It is the lowest level that offers IP-based transport over wired and wireless access technologies ranging from LAN, DSL, UMTS, DVB, and WiMax. All these access technologies should enable unicast, multicast and broadcast transmission with QoS support and seamless service mobility.

Media Delivery Plane composed a distributed set of media processor called Media Delivery Function Processor (MDFP) that performs media processing such as streaming, transcoding, and recording function and relay media stream from Content Provider (CP) to consumers over IP-based unicast, multicast or broadcast bearers. The MDF is placed as a mediator between the CP and the Access and Transport Plane. It is introduced as an evolved Media Resource Function in IMS architecture. Besides, it is responsible for resource scheduling, content distribution, congestion control and content adaptation.

Control Plane: This plane is on the top of the access and delivery planes and based on the IMS core that includes the Call Session Control Functions (CSCF), the Home Subscriber Function (HSS) and gateway to the legacy networks. This plane is responsible for session control and service triggering on the service enabler layer based on defined filter criteria stored for each user on the HSS. Furthermore, the Media Delivery Function Controller (MDFC) is in charge of controlling the MDFPs relied on requests triggered by the service enablers. Based on the capabilities of the end-terminal as well as access network - among other factors - the MDFC selects a MDFP to perform the relevant media processing functions.

Service Enabler Plane: This plane poses the essential functions that enable the interworking between the service provider, the network operator and the end-user. It contains several enablers that each offers intrinsic and elementary function. Therefore this plane performs all related control functions among these four actors and makes use of all capabilities exposed by the media delivery plane. The design of this plane follows the OMA approach and OMA Service Environment that have been mentioned in section 2.5. Design and development issues of this plane can be found in more details in [10].

Application Plane: This plane hosts services that utilize from the basic functions offered on the Service Enabler Plane through open and policy-based standardized interfaces such as Parlay $\mathrm{X}$. Services on this plane are deployed as standalone service or hosted in an application server. Such application provides a base for the validation of underlying entities. Sophisticated application development tools and Model Driven Architecture (MDA) tools provide a highly comfortable way to develop services.

User Premises Plane: This plane presents all possible interconnected access technologies either wired or wireless and enables the user to get IP connectivity directly or via an intermediate gateway hosted at user premises. This gateway offers the user the ability to build up a meshed network within his home environment. Every network capable device is connected to this gateway to offer incorporation with other local devices or connect to the core network. Examples are IPTV clients, VoIP client, SetTop-Boxes (STB), PCs and smart IP devices to control the digital home. It communicates with the IMS core through the $\mathrm{Gm}, \mathrm{Mb}$ and Ut reference points based on SIP, RTP/RTCP and SHTTP, respectively.

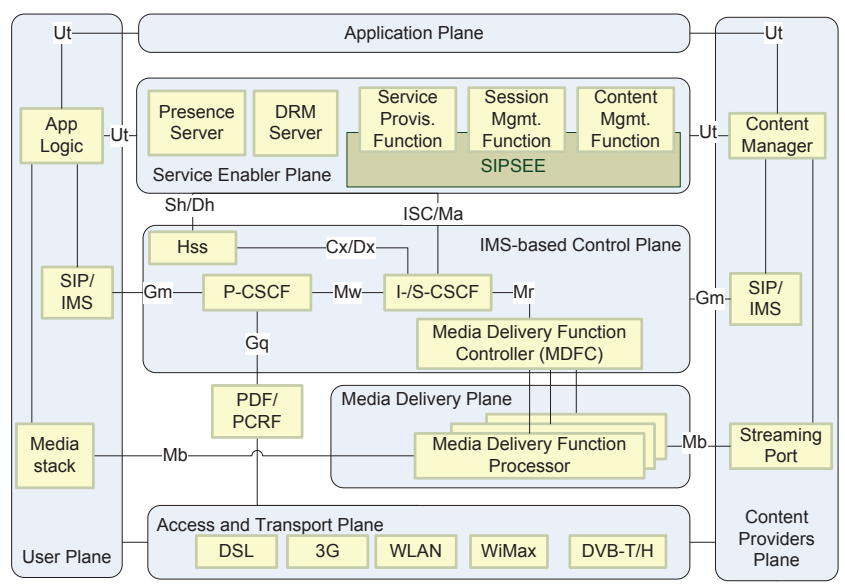

\section{Figure 3: The FOKUS 3Gb/NGN Testbed Architecture}

Content Provider Plane: This plane presents the content provider that produces, edits and offers multimedia content to the service provider. Therefore it is the sources of all types of multimedia content. To enable end-user to behave as a CP this plane makes use of all reference points $(\mathrm{Gm}, \mathrm{Mb}$, and $\mathrm{Ut})$ that are defined between the end-terminal and the network side.

\section{APPLICATION SCENARIOS AND VALIDATION WITH THE FOKUS NGN/IMS}

The service enabler plane of the MIL architecture includes several service enablers that allow developing more intelligent application easily. Part of these enablers as well as applications are developed and deployed on the lab as standalone solution or hosted on an Application Server (AS). FOKUS has developed several types of application servers that are deployed at FOKUS infrastructure [12]. The SIP Servlet Execution Environment (SIPSEE) [13] is one of these AS as a SIP server and is based on the SIP Servlet technology. Demo-applications are used for demonstration purposes and for validation of concepts and software components through the real life.

The next two subsections describe the reference architecture of the implementation and the sequence diagram of the session setup of a live TV or linear TV session based on IMS signalling with its related measurement values.

\subsection{Reference Implementation}

Based on the logical architecture discussed in the previous section we developed a prototype of such framework that enables provisioning of multimedia streaming Services with interactivity and more mobility features over unicast, multicast and broadcast access network. This solution has been deployed in the lab (MIL) and can be considered as proof of concept for FOKUS IMS-based triple play architecture discussed in the last section. 
The ability of delivering triple play services over unicast (UMTS), multicast (LAN/WLAN) and broadcast (DVB-T/H) access networks and through the IMS raises the possibility to combine the advantages of DVB-T/H for delivering streaming services with high quality at the same time the UMTS for enabling personalization and interactivity. Furthermore IMS core for secure, chargeable, personalized, and interactive transfers. Furthermore IMS enables seamless integration of these access networks with quality of service and mobility support.

On the SIPSEE a Session Management Enabler (SME) and a Content Manager Enabler (CME) are deployed. The SME performs all related service control and session management. The SME is charge of session setup of live TV or Video-on-Demand content, bearer selection, mobility across several access networks, group management and trigger the media delivery plane for content delivery by performing the relevant media processing. The CME is in charge of session setup to and from content providers and distribute the content into the media delivery plane. The Service Provisioning Functions (SPF) provides consumers with the information related to service provisioning and enables the consumer to discover the available services or content. The Presence Server manages and maintains the presence information of all consumers. With the context of triple play consumer may share not only his availability information with his buddies but they can announce his current activity (e.g. the TV channel, gaming, etc.)

The IMS core is based on the FOKUS open sources implementation of the P-/I-S-CSCF and the HSS [11]. The media delivery plane is realized through a set of distributed media servers that provide media processing and content delivery from the content provider to the consumers. Each media server called Media delivery Function Processor (MDFP) can be considered as an enhanced Media Resource Function Processor (MRFP) defined in IMS specifications, whereas the improvement tackle the video processing tasks like transcoding, streaming and recording. Furthermore the MDFP can be controlled by the SME and the CME via SIP to play and relay content from a unicast or multicast input to a unicast, multicast or broadcast output.

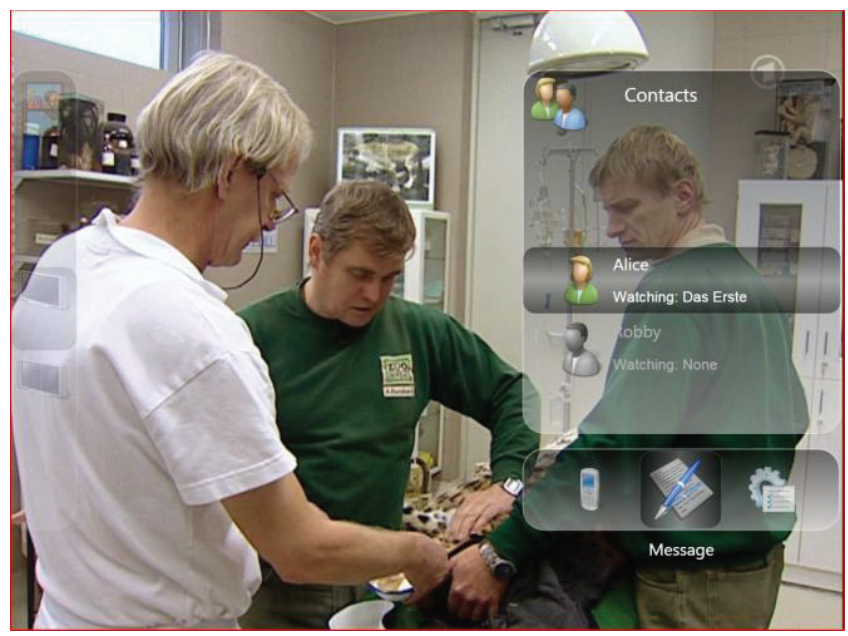

Figure 4: NGN Multimedia Client

On the user side an advance multimedia client is developed for Windows operating system and based on .NET that makes use of all features provided by the network entities through the $\mathrm{Gm}, \mathrm{Mb}$ and Ut reference points defined in the IMS specifications. The client is facilitated for TV services (live TV and VoD), telecommunication services (telephony, chat, messaging and presence) as well as cross fertilization services such as displaying incoming call, pause the stream of a VoD movie or mute the tone of live TV during a call, watch what your friends currently watching and session mobility of TV session. Figure 5 shows the GUI of the corresponding client.

\subsection{Signaling}

In this section, the live TV scenario is selected to illustrate the benefits of the IMS-based framework. This scenario enables the end user to watch live TV channel. Since TV channels are consumed by a mass of users the next sequence diagram illustrates session setup for a multicast bearer instead of a unicast bearer for content delivery. For this reason the SIP Prack and Update methods are not used for triggering and allocating the QoS, but the SIP Invite method is used to carry out the channel request and terminal capabilities to the SMF.

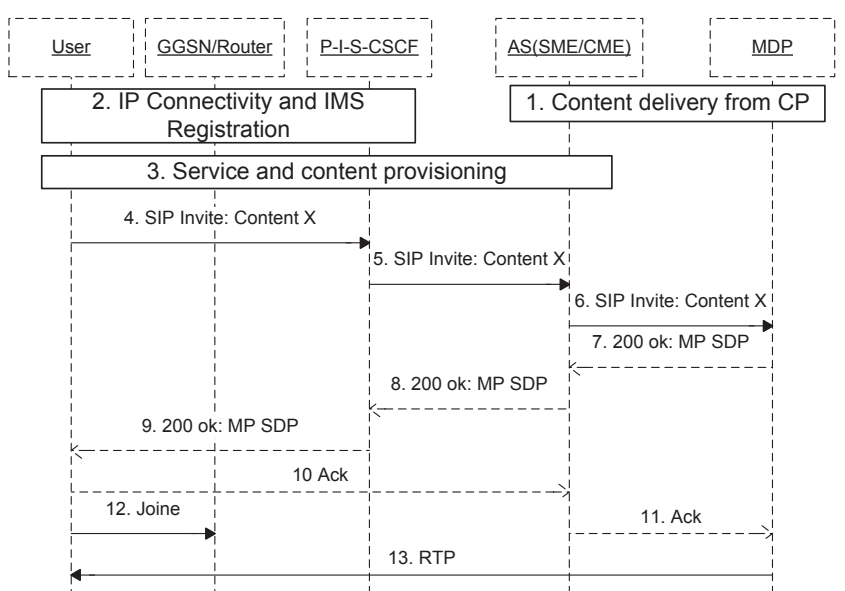

Figure 5: Sequence flows of Live TV session setup

In the first phase, we assume that the content is already delivered from the $\mathrm{CP}$ to the MDCF/MDFP. After the user got IP connectivity and registered with the IMS core, he receives provisioning information for all subscribed services and the related resources (URLs). Figure 6 shows the sequence flows when the session is setup.

When he/she selects a particular channel the terminal initiates a SIP Invite message with the URL of the corresponding channel and terminal capabilities. The IMS core (P-/I-/S-CSCF) routs the request to the SME on the SIPSEE AS based on the filter criteria stored in user's profile. The SME decides for a multicast bearer for content delivery and consequently it behaves as a SIP back-toback user agent to trigger the MDF for relaying the related content stream to an allocated IP multicast address. Though this requests is sent to the MDFC/MDFP only once and independent of following users' requests received by the SME. The MDFC/MDFP responds with a 200 ok message with the corresponding session parameters such as supported codecs and ports. Accordingly the SME generates a 200 ok message including session parameters and forwards it to the IMS core, which forwards the message to the UE. 
Once the UE receives the 200 ok message it activates the multicast bearer by sending a Joint message with the corresponding multicast address to the IP core (e.g. to the GGSN in UMTS or the router in LAN or WLAN). The access and IP core may start allocating the corresponding resources for this session. In fact we have not tackled this problem in our implementation yet. Then the UE sends a SIP ACK back to the SME which creates a SIP ACK to the MDF to start the transmission.

We have conducted several measurement of an end-to-end session setup for live TV session based on our implementation and using to access networks, namely fast-Ethernet LAN and WLAN $802.11 \mathrm{~g}$ with a theoretical bandwidth of $100 \mathrm{Mbps}$ and $54 \mathrm{Mbps}$, respectively. The IMS core, the SME and MDFP are deployed on a fast-Ethernet LAN. The measurement is designed to determine the signaling delay from sending a SIP Invite message up to receiving the 200 OK message by the UE (UE $\leftarrow \rightarrow$ IMS core $\leftarrow \rightarrow$ SME on the SIPSEE $\leftarrow \rightarrow$ MDF).

All results base on the statistical average of 20 record sets. Table 1 shows the statistical average value $\mu$ and the deviation $\sigma$.

\begin{tabular}{|l|l|l|}
\hline Access & $\mu$ in $\mathrm{ms}$ & $\sigma$ in $\mathrm{ms}$ \\
\hline LAN & 76 & 10 \\
\hline WLAN $(802.11 \mathrm{~g})$ & 84 & 30 \\
\hline
\end{tabular}

Table 1: End-to-End signaling delay

The signalling delay can be considered from two perspectives. The first perspective addresses the access network. Due to low load of signalling traffic on the RAN there is so far no significant difference between LAN and WLAN regarding the signalling delay. However, the values concerning the WLAN access are quite variant regarding the standard deviation.

Based on these results, it is obvious that IMS can be considered not only for telecommunication services but it can be regard as a basic control framework for delivering multimedia services including TV, voice and data services over unicast, multicast and broadcast networks.

\section{CONCLUSION AND FURTHER WORK}

In this paper we presented the FOKUS Media Interoperability Lab as a show room for a step towards converged triple play architecture. The described lab facilitates the delivery of enriched multimedia content over unicast, multicast or broadcast access networks. The functionalities of the corresponding architecture were split among several logical planes and entities to shape a flexible, context aware and QoS supporting end-to-end design.

Moreover, the IMS signalling was extended in order to enable IMS with multicast and broadcast capabilities. Finally, the up to now unspecified interface to the $\mathrm{CP}$ was taken into consideration.

Finally the conducted measurement results show that is evident that IMS can be considered for delivering not only telecommunication service, but also TV services.

Future work will be dedicated to develop other buildings blocks including service provisioning enabler, interactivity manager and personalization manager. Furthermore QoS issues for unicast bearers will be taken into consideration.

\section{REFERENCES}

[1] 3GPP, TS 23.228. IP Multimedia Subsystem; (stage 2). www.3gpp.org

[2] http://www.3gpp2.org

[3] DTR 00001, Telecommunications and Internet Converged Services and Protocols for Advanced Networking (TISPAN); Release 1 Definition.

[4] 3GPP TS 23.246: "Multimedia Broadcast/Multicast Service; Architecture and Functional Description", Release 6

[5] ETSI TISPAN ES2020, (2005) "Resource Admission Control Subsystem (RACS) Architecture".

[6] Yang Li and H. Anthony Chan, "Design of Network Intelligence for Future Generation Network on an IP Multimedia Subsystem Testbed," Information Society Technologies in Africa (IST-Africa), Pretoria, South Africa, 3-5 May 2006.

[7] http://uctimsclient.berlios.de/

[8] ETSI TS 102 034, Digital Video Broadcasting (DVB); Transport of MPEG2 Based DVB Services over IP Based Networks

[9] Adel Al-Hezmi; Oliver Friedrich; Stefan Arbanowski; Thomas Magedanz; "Requirements for an IMS-based Quadruple Play Service Architecture"; Network, IEEE; Volume 21, Issue 2, March-April 2007 Page(s):28 - 33; Digital Object Identifier 10.1109/MNET.2007.334309

[10] A. Al-Hezmi, B. Mrohs, C. Räck, M. Elkotob, Stephan Steglich; "Next Generation Service Architecture: Challenges and Approaches", IEEE Applications and Services in Wireless Networks (ASWN) 29-31 May 2006, http://www.aswn2006.org/, Berlin, Germany

[11] D. Vingarzan, P. Weik, T. Magedanz: "Development of an open source IMS core for emerging IMS testbeds", Special Issue on IMS, Journal on Mobile Multimedia (JMM), Vol.2 No., Rinton Press, Princeton, USA, 2006, http://www.rintonpress.com/journals/jmm/

[12] K. Knuettel, T. Magedanz, L. Xie: "SIP Servlet Execution Environment (SIPSee) - An approved IMS SIP Application Server for Converged Applications", International Conference on Intelligence in Networks (ICIN) 2006 , Bordeaux, France, May 31, 2006, http://www.icinconference.com/ 